\title{
Differences in Outcomes between Early and Late Diagnosis of Cystic Fibrosis in the Newborn Screening Era
}

\author{
Michael J. Coffey, BMed, MD ${ }^{1}$, Viola Whitaker ${ }^{1}$, Natalie Gentin, MBBCh, FRACP ${ }^{1,2}$, Rosie Junek, DCH ${ }^{3}$, \\ Carolyn Shalhoub, BSc(Hons), GradDip(Counselling) ${ }^{4}$, Scott Nightingale, BMed(Hons), MClinEpid, FRACP ${ }^{5,6}$, \\ Jodi Hilton, BMed, FRACP ${ }^{7,8}$, Veronica Wiley, PhD ${ }^{3,9}$, Bridget Wilcken, MD, FRACP ${ }^{3,9}$, Kevin J. Gaskin, MD, FRACP ${ }^{3,9}$, and \\ Chee Y. Ooi, MBBS, FRACP, PhD ${ }^{1,10}$
}

Objectives To evaluate children with cystic fibrosis (CF) who had a late diagnosis of CF (LD-CF) despite newborn
screening (NBS) and compare their clinical outcomes with children diagnosed after a positive NBS (NBS-CF). Study design A retrospective review of patients with LD-CF in New South Wales, Australia, from 1988 to 2010 was performed. LD-CF was defined as NBS-negative (negative immunoreactive trypsinogen or no F508del) or NBSpositive but discharged following sweat chloride $<60 \mathrm{mmol} / \mathrm{L}$. Cases of LD-CF were each matched 1:2 with patients with NBS-CF for age, sex, hospital, and exocrine pancreatic status.

Results A total of 45 LD-CF cases were identified (39 NBS-negative and 6 NBS-positive) with 90 NBS-CF matched controls. Median age (IQR) of diagnosis for LD-CF and NBS-CF was $1.35(0.4-2.8)$ and $0.12(0.03-0.2)$ years, respectively $(P<.0001)$. Estimated incidence of LD-CF was 1 in 45000 live births. Compared with NBS-CF, LD-CF had more respiratory manifestations at time of diagnosis $(66 \%$ vs $4 \% ; P<.0001)$, a higher rate of hospital admission per year for respiratory illness $(0.49$ vs $0.2 ; P=.0004)$, worse lung function (forced expiratory volume in 1 second percentage of predicted, 0.88 vs $0.97 ; P=.007)$, and higher rates of chronic colonization with Pseudomonas aeruginosa ( $47 \%$ vs $24 \%$; $P=.01$ ). The LD-CF cohort also appeared to be shorter than NBS-CF controls (mean height $z$-score -0.65 vs $-0.03 ; P=.02$ ).

Conclusions LD-CF, despite NBS, seems to be associated with worse health before diagnosis and worse later growth and respiratory outcomes, thus providing further support for NBS programs for CF. (J Pediatr 2017;181:137-45).

ystic fibrosis $(\mathrm{CF})$ is a life-shortening recessive disorder, caused by mutations in the $C F$ transmembrane conductance regulator (CFTR) gene, which affects approximately 1 in 3000 newborns in Caucasian populations. ${ }^{1-7} \mathrm{CF}$ is now commonly diagnosed via newborn screening (NBS) in many countries, ${ }^{8}$ with the state of New South Wales (NSW) in Australia implementing screening in July 1981. Screening in NSW initially began with a 2-tier immunoreactive trypsinogen (IRT) protocol, with dried blood spots collected on days 3-5 (subsequently changed to days 2-4) of life and again at 4-6 weeks if increased. ${ }^{9}$ In 1993 NSW changed to an IRT-DNA system: IRT level > 99th percentile and F508del constituting a positive test. Testing during this period was for the F508del mutation only (which was at the time present as either one or 2 copies in 94\% of NSW patients with $\mathrm{CF}^{10}$ ). Dependent on whether F508del was found on 1 or both alleles, referral to a Cystic Fibrosis Clinic for treatment, sweat testing, and possibly further genotyping was recommended.

In 2009 a Cochrane review of NBS for CF highlighted that the Wisconsin NBS trial was the only one meeting randomized, controlled trial criteria. ${ }^{11}$ This review concluded that severe malnutrition was less common among screened babies ${ }^{12}$ and the NBS provided potential for better respiratory outcomes ${ }^{13}$; however, the later

$\begin{array}{ll}\text { BMI } & \text { Body mass index } \\ \text { CF } & \text { Cystic fibrosis } \\ \text { CFSPID } & \text { CF screen positive inconclusive diagnosis } \\ \text { CFTR } & \text { CF transmembrane conductance regulator } \\ \text { FEV1\% } & \text { Forced expiratory volume in 1 second percentage of predicted } \\ \text { IRT } & \text { Immunoreactive trypsinogen } \\ \text { LD-CF } & \text { Late diagnosis of cystic fibrosis } \\ \text { LD-NBS-neg } & \text { Late diagnosis newborn screen negative } \\ \text { LD-NBS-pos } & \text { Late diagnosis newborn screen positive } \\ \text { MI } & \text { Meconium ileus } \\ \text { NBS } & \text { Newborn screening } \\ \text { NBS-CF } & \text { Newborn screen diagnosed CF } \\ \text { NSW } & \text { New South Wales } \\ \text { PI } & \text { Pancreatic insufficient } \\ \text { PS } & \text { Pancreatic sufficient } \\ \text { SC } & \text { Sweat chloride }\end{array}$

From the ${ }^{1}$ School of Women's and Children's Health, Faculty of Medicine, University of New South Wales, Sydney, Australia; ${ }^{2}$ Department of General Pediatrics, Sydney Children's Hospital Randwick, Sydney, Australia; 3The Children's Hospital at Westmead, Sydney, Australia; ${ }^{4}$ Department of Medical Genetics, Sydney Children's Hospital Randwick, Sydney, Australia; ${ }^{5}$ GrowUpWell Priority Research Centre, University of Newcastle, Newcastle, Australia; ' ${ }^{6}$ Department of Gastroenterology, John Hunter Children's Hospital, Newcastle, Australia; ${ }^{7}$ University of Newcastle, Newcastle, Australia; ${ }^{8}$ Department of Respiratory Medicine, John Hunter Children's Hospital, Newcastle, Australia; ${ }^{9}$ The University of Sydney, Sydney, Australia; and ${ }^{10}$ Department of Gastroenterology, Sydney Children's Hospital Randwick Sydney, Australia

The authors declare no conflicts of interest.

0022-3476/\$ - see front matter. @ 2016 Elsevier Inc. All rights reserved.

http://dx.doi.org10.1016/j.jpeds.2016.10.045 
findings were confounded by a high infection rate of Pseudomonas aeruginosa. The prolonged debate over the benefit of NBS has been largely due to the lack of limited randomized, controlled trial data. Although good quality evidence was available, limitations often included the retrospective nature and selection criteria of studies. ${ }^{14-21}$ Beyond those diagnosed by NBS, the outcomes of those who are missed by NBS are unknown. The first attempt to look for missing cases was undertaken by Massie et al in 2000. ${ }^{22}$ In Australian populations, the IRT-DNA protocol has performed with a sensitivity of $94 \%$ and a specificity of $99.9 \%,{ }^{10}$ which is comparable with other US and European centers. ${ }^{8}$ Accounting for differences in IRT cutoffs and the number of mutations in DNA panels worldwide, the sensitivity of NBS varies between $86.6 \%$ and $97.5 \%{ }^{8}$ To date, there are no publications specifically analyzing those patients with a false-negative NBS result.

Interest in the subset of patients with an inconclusive diagnosis of CF after NBS has emerged, with the terms "CFTRrelated metabolic syndrome" 23 and "CF screen positive inconclusive diagnosis" (CFSPID) ${ }^{24,25}$ proposed by US and European groups, respectively. In a prospective study, approximately $11 \%$ of infants with an initial inconclusive diagnosis will subsequently receive a diagnosis of $\mathrm{CF}$, thus, arguing the need for follow-up of this population.

Due to the opportunity for early intervention and preventative care of patients with CF, data about those with a missed diagnosis are important. These data includes incidence, characteristics (including genotype and phenotype), and longterm outcomes. Given the long history of NBS in NSW, we are provided with a unique opportunity to evaluate this subset of patients. Our study aimed to provide observations from the important group of patients with CF who had a late diagnosis of CF (LD-CF) despite NBS, and determined whether their clinical outcomes differ from children diagnosed with CF after a positive NBS (newborn screen diagnosed CF [NBS-CF]) during the same time period.

\section{Methods}

A retrospective review of patients with LD-CF from April 1988 (commencement of the Australian CF Data Registry) to December 2010 within NSW was performed. All cases with LDCF were identified through the Australian CF Data Registry, CF clinics (Sydney Children's Hospital Randwick, Children's Hospital Westmead, and John Hunter Children's Hospital), and the NSW NBS Programme. This study was approved by the human research ethics boards of all participating institutions (LNR/11/SCHN/310).

Patients were included if they met the diagnostic criteria for CF and were defined as LD-CF (as per definitions below). Patients were excluded if they (1) did not meet the diagnostic criteria for CF, (2) did not receive NBS, (3) were born before April 1988, from outside NSW or there was insufficient data for any other reason, or (4) were not diagnosed late.
As a comparator, each subject with LD-CF was matched 1:2 with patients with NBS-CF for age, sex, exocrine pancreatic status (determined by either the coefficient of fat absorption from 72-hour fecal fat collection and/or fecal elastase), and CF clinic (ie, same clinic) using the Australian CF Data Registry. If multiple matches were identified, the control with the smallest age difference to the subject was selected. If pancreatic function status was unknown for the subject, they were matched with pancreatic insufficient (PI) controls. Demographic, clinical (including calculated $z$-scores for growth variables), and laboratory data were collected from the aforementioned databases.

During the study period (April 1988-December 2010), the NBS protocol in NSW included a dried blood spot IRT level for all newborns. From April 1988 to March 1993, those patients who had an elevated IRT (top $0.7 \%$ and/or $>100 \mu \mathrm{g} / \mathrm{L}$ whole blood) had a repeat IRT performed and if the second was also elevated ( $>75 \mu \mathrm{g} / \mathrm{L}$ whole blood), they would subsequently undergo sweat testing. Mutation screening for F508del began in April 1993 and those with an elevated IRT (top 1\% and/or $>75 \mu \mathrm{g} / \mathrm{L}$ whole blood) would undergo F508del mutation analysis. If F508del was identified, either 1 or 2 copies, sweat testing, and, at the discretion of treating clinician, an extended genetic mutation panel was performed.

CF as defined by the US CF Foundation Consensus Report ${ }^{20}$ consists of $\geq 1$ characteristic phenotypic feature(s) of disease plus sweat chloride (SC) $\geq 60 \mathrm{mmol} / \mathrm{L}$, and/or identification of CF disease-causing mutations on both alleles. Alternatively in the absence of symptoms, a diagnosis of CF in a sibling is sufficient as phenotypic criteria. Mutations were classified as disease-causing using the CFTR2 project. ${ }^{26,27}$

LD-CF was defined as an individual who fulfilled the criteria for CF and was either (1) NBS negative (LD-NBS-neg) with a negative IRT or genotype (ie, no F508del identified) or (2) NBS positive (LD-NBS-pos) but discharged after a $\mathrm{SC}<60 \mathrm{mmol} / \mathrm{L}$.

Chronic Pseudomonas aeruginosa infection was defined by 3 consecutive respiratory cultures within a 12 -month period and/or isolation of mucoid P aeruginosa. ${ }^{28}$ Chronic Staphylococcus aureus infection was defined by 3 consecutive positive respiratory cultures within a 12-month period.

\section{Statistical Analyses}

Case-control matching analysis of age (on December 31, 2010) was performed using Pearson correlation to assess effectiveness of matching. Cohort analysis was performed with comparisons between LD-CF (including LD-NBS-neg and LDNBS-pos subsets) and NBS-CF cohorts made by utilizing Student $t$ test or Mann-Whitney $U$ test for continuous variables (presented as mean [SD] and median [IQR] for normally and not normally distributed data, respectively) and by Fisher exact test for categorical variables. Linear mixed models analysis was utilized to assess clinical measurements performed throughout the study period and is presented as mean (SD). $P<.05$ was considered statistically significant. All statistical calculations and graphs were performed in SPSS 22.0 (SPSS Inc, Chicago, Illinois). 


\section{Results}

A total of $45 \mathrm{LD}-\mathrm{CF}$ cases were identified and included along with 90 matched NBS-CF controls. Of the initial 68 LD-CF cases identified, 4 did not meet the diagnostic criteria for $\mathrm{CF}$, 1 did not receive NBS, 1 was born before April 1988, 1 was born outside NSW, 1 moved from NSW, 4 were lost to follow-up, 1 was a duplicate patient, 3 patients with CF had meconium ileus (MI), and 7 patients with NBS-CF in whom there was a documented reason for delay in performing a sweat test and/ or the diagnosis of CF was not late.

The demographic data for LD-CF and patients with NBSCF are summarized in Table I. The estimated incidence of CF in NSW during the study period was 1 in 2200 live births, whereas the incidence of LD-CF was 1 in 45000 live births. Thus, approximately 1 in every 21 patients with CF were LDCF. The LD-CF and patients with NBS-CF were matched for sex, hospital location, exocrine pancreatic sufficiency status, and significantly matched for age (Pearson correlation, $P<.0001)$. Median age (IQR) of diagnosis for LD-CF and NBSCF was 1.35 years $(0.36-2.81)$ and 0.12 years $(0.03-0.16)$ respectively $(P<.0001)$.

Of the LD-CF cases, 39 were LD-NBS-neg (22 negative IRT and 17 negative genotype for F508del) and 6 were LD-NBSpos (2 with a SC $<30 \mathrm{mmol} / \mathrm{L}$ and 4 with a SC $30-60 \mathrm{mmol} /$ L) (Figure 1; available at www.jpeds.com).

\section{Genotypic and Phenotypic Data}

The genotypic and phenotypic data for each patient with LDCF and NBS-CF-matched control is listed in Table II. Almost one-half of the patients with LD-CF (49\%) had a negative IRT < 99th percentile. Of the patients with LD-CF, 31 (69\%) had CFTR mutations identified on both alleles, 8 (18\%) had only 1 mutation identified, and 6 (13\%) had no mutations

\begin{tabular}{|c|c|c|c|}
\hline Characteristics & LD-CF $(n=45)$ & NBS-CF $(n=90)$ & Matching \\
\hline \multicolumn{4}{|l|}{ Age (years) } \\
\hline At diagnosis* & $1.35(0.36-2.81)$ & $0.12(0.03-0.16)$ & $P<.0001$ \\
\hline At $31 / 12 / 2010$ & $9.73(0.84)$ & $10.02(0.59)$ & $P<.0001^{\dagger}$ \\
\hline \multicolumn{4}{|l|}{ Sex } \\
\hline Male & 24 & 48 & $100 \%$ \\
\hline Female & 21 & 42 & $100 \%$ \\
\hline \multicolumn{4}{|l|}{ Hospital } \\
\hline CHW & 21 & 42 & $100 \%$ \\
\hline $\mathrm{JHCH}$ & 7 & 14 & $100 \%$ \\
\hline $\mathrm{SCH}$ & 17 & 34 & $100 \%$ \\
\hline \multicolumn{4}{|c|}{ Pancreatic function ${ }^{\ddagger}$} \\
\hline $\mathrm{PI}$ & 27 & 54 & $100 \%$ \\
\hline PS & 10 & 20 & $100 \%$ \\
\hline Unknown ${ }^{\S}$ & 8 & $16(\mathrm{Pl})$ & - \\
\hline
\end{tabular}

$\mathrm{CHW}$, Children's Hospital at Westmead; JHCH, John Hunter Children's Hospital; SCH, Sydney Children's Hospital Randwick.

Data presented as median (IQR) or mean (SD).

${ }^{\star}$ Age at diagnosis significantly different ${ }^{*} P<.0001$.

$\dagger$ Age at December 31, 2010 (for matching analysis), significantly effective using Pearson correlation. $\ddagger$ Pancreatic function determined by the coefficient of fat absorption from 72-hour decal fat collection and/or fecal elastase-1.

$\S$ Cases with unknown pancreatic function status matched with PI controls. identified on either allele. Two disease-causing mutations (ie, not including those with varying clinical consequence or unknown significance) were identified in 23 (51\%) and 72 $(80 \%)$ of patients with LD-CF and NBS-CF, respectively $(P=.001)$. SC levels were not significantly different between patients with LD-CF and patients with NBS-CF with the mean (SD) initial SC values being 83.2 (4.4) and 89.1 (2.1) respectively $(P=.2)$. Pancreatic function status was known in 37 of the patients with LD-CF (82\%), and of those, 27 (73\%) were PI and 10 (27\%) were pancreatic sufficient (PS). The rate of PI was significantly higher in the LD-NBS-neg cohort compared with the LD-NBS-pos cohort ( $81 \%$ vs 20\% respectively; $P=.01$ ).

\section{Clinical Data}

At the time of diagnosis, 38 patients with LD-CF (84\%) and 90 patients with NBS-CF (100\%) had manifestations of CF recorded. None of the LD-CF cohort had MI; MI was recorded in $12 \%$ of the NBS-CF cohort $(P=.02)$. The LD-CF cohort presented with significantly more respiratory (66\% vs $4 \%$; $P<.0001)$, gastrointestinal (24\% vs $6 \% ; P=.005)$, and failureto-thrive manifestations $(29 \%$ vs $3 \% ; P<.0001)$ when compared with NBS-CF controls at the time of diagnosis. Subset analysis revealed both LD-NBS-neg ( $54 \%$ vs $4 \%$; $P<.0001$ ) and LD-NBS-pos ( $67 \%$ vs $8 \% ; P=.02)$ cohorts to have significantly more respiratory manifestations than matched patients with NBS-CF.

Hospitalization, clinical, and laboratory data were recorded during follow-up at CF clinics, with data over a median of $6.5(3.0-9.0)$ and $8.0(3.0-11.0)$ years for the LD-CF $(\mathrm{n}=38)$ and NBS-CF $(\mathrm{n}=88)$ cohorts respectively $(P=.3)$. The median number of hospital admissions per year for CF-related respiratory illness was significantly higher for patients with LD-CF compared with patients with NBSCF (0.49 [0.2-1.1] vs 0.2 [0-0.5] respectively; $P=.0004)$; it was also significantly higher for the LD-NBS-neg subset compared with patients with NBS-CF (0.44 [0.2-1.0] vs 0.2 [0-0.5] respectively; $P=.001)$. The total number of hospital days for CF-related respiratory illness was also significantly higher for patients with LD-CF compared with patients with NBS-CF (25.0 [10.5-69.3] vs 5.0 [0-23.0], respectively; $P=.001)$ and patients who were LD-NBS-neg compared with patients with NBS-CF (25.0 [10.0-83.0] vs 7.0 [0-29.0] respectively; $P=.004)$. There were fewer hospital admissions per year and no difference between the LD-CF and NBS-CF cohorts in regards to CF-related gastrointestinal illness $(0$ [0-0.03] and 0 [0-0], respectively; $P=.5)$ and other CF-related illness requiring admission $(0[0-0.2]$ and $0[0-0.1]$, respectively; $P=.2)$.

All growth variables and lung function testing results were analyzed over the duration of follow-up using linear mixed models (Figure 2). The mean (SD) height $z$-score was significantly lower for patients with LD-CF compared with NBSCF $(-0.65[0.22]$ vs $-0.03[0.15] ; P=.02)$. No difference between the LD-CF and NBS-CF cohorts in regard to mean (SD) $z$-scores for weight $(-0.48[0.21]$ vs 0.01 [0.14], respectively; $P=.06)$ or body mass index (BMI) $(-0.11[0.17]$ vs -0.03 
Table II. Genotype and phenotype of patients with LD-CF with matched NBS-CF controls (1:2)

\begin{tabular}{|c|c|c|c|c|c|c|}
\hline & PF & Initial (peak) SC & NBS-CF 1 & SC & NBS-CF 2 & SC \\
\hline \multicolumn{7}{|l|}{ NBS Negative (LD-NBS-neg) } \\
\hline \multicolumn{7}{|l|}{ IRT Negative } \\
\hline F508del/F508del & $\mathrm{PI}$ & 112 & F508del/F508del & - & F508del/F508del & - \\
\hline F508del/F508del* & $\mathrm{Pl}$ & 102 & F508del/F508del & 81 & F508del/G542X & 110 \\
\hline F508del/F508del* & $\mathrm{PI}$ & 82 & F508del/F508del & 100 & F508del/F508del & 106 \\
\hline F508del/F508del* & $\mathrm{PI}$ & 82 & F508del/F508del & 80 & F508del/Unknown & 82 \\
\hline F508del/F508del & PS & 112 & F508del/F508del & - & F508del/Unknown & 70 \\
\hline F508del/G551D & $\mathrm{PI}$ & 96 & F508del/Q493X & - & F508del/2789 + 2insA & 67 \\
\hline F508del/1717-1G > A & $\mathrm{Pl}$ & 109 & F508del/F508del & 105 & F508del/F508del & 60 \\
\hline F508del/G542X & $\mathrm{Pl}$ & 80 & F580del/F508del & 92 & F508del/F508del & - \\
\hline F508del/R1162X & - & 104 (108) & F508del/F508del & - & F508del/c.2252G $>T$ & 74 \\
\hline F508del/R117H & PS & 78 & F508del/Unknown & 105 & F508del/Unknown & 70 \\
\hline 3659delC/621 + 1G > T & $\mathrm{PI}$ & 93 & F508del/F508del & 75 & F508del/Unknown & 105 \\
\hline Q493X/2789 + 2insA & - & 78 & F508del/F508del & - & F508del/F508del & 92 \\
\hline$R 117 H \overline{R 117 H}$ & PS & $27(68)$ & F508del/L206W & 45 & F508del/F508del & 92 \\
\hline F508del/Unknown* & $\mathrm{PI}$ & 124 & F508del/F508del & 97 & F508del/F508del & 90 \\
\hline F508del/Unknown & $\mathrm{PI}$ & 98 & F508del/Unknown & 85 & F508del/F508del & 128 \\
\hline F508del/Unknown* & $\mathrm{Pl}$ & 96 & F508del/F508del & 94 & F508del/F508del & - \\
\hline F508del/Unknown & PS & $29(65)$ & F508del/Unknown & 63 & F508del/R117H & 86 \\
\hline R117H/Unknown & PS & $34(62)$ & F508del/Unknown & 78 & F508del/1717-1G > A & 97 \\
\hline R117H/Unknown & PS & $49(67)$ & F508del/Unknown & 96 & F508del/F508del & - \\
\hline Unknown/Unknown* & PI & 104 & F508del/F508del & 99 & F508del/F508del & 121 \\
\hline Unknown/Unknown & - & 78 & F508del/3659delc & 84 & F508del/Unknown & 73 \\
\hline Unknown/Unknown & - & $40(64)$ & Unknown/Unknown & 95 & F508del/F508del & 110 \\
\hline \multicolumn{7}{|l|}{ F508de/ Negative } \\
\hline G551D/V520F & $\mathrm{Pl}$ & 105 & F508del/G551D & 72 & F508del/F508del & 86 \\
\hline G551D/1525-1G > A & $\mathrm{Pl}$ & 120 & F508del/G551D & 85 & F508del/F508del & 74 \\
\hline G551D/N1303K & $\mathrm{Pl}$ & 98 & F508del/N1303K & 132 & F508del/F508del & 81 \\
\hline E822X/E822X & $\mathrm{Pl}$ & 102 & F508del/F508del & 92 & F508del/F508del & 88 \\
\hline 2183AA $>$ G/ & $\mathrm{Pl}$ & 88 & F508del/F508del & 115 & F508del/F508del & - \\
\hline \multicolumn{7}{|l|}{ 2183AA $>$ G } \\
\hline $621+1 G>T / M 1 V$ & $\mathrm{Pl}$ & $80(98)$ & F508del/Unknown & 92 & 3659delC/621 + 1G > T & 93 \\
\hline R1066C/1154insTC & $\mathrm{Pl}$ & 102 & F508del/G542X & 100 & F508del/W1282X & 105 \\
\hline R1066C/1154insTC & - & 102 & F508del/394delTT & - & F508del/F508del & 97 \\
\hline S4X/S4X & $\mathrm{Pl}$ & 107 & F508del/F508del & 90 & F508del/F508del & - \\
\hline c. $3386-2 A>G / c .3469-65 C>A$ & $\mathrm{Pl}$ & 90 & F508del/F508del & 98 & F508del/W846X & 90 \\
\hline G551D/Unknown & PI & 121 & F508del/1717-1G > A & 103 & F508del/F508del & 86 \\
\hline E822X/Unknown & $\mathrm{PI}$ & 114 & F508del/F508del & 96 & F508del/G551D & 85 \\
\hline $621+1 G>T /$ Inknown & - & 93 & F508del/E60X & - & F508del/F508del & - \\
\hline c.3890_3891inst/Unknown & - & 106 & F508del/F508del & 78 & F508del/G542X & 100 \\
\hline Unknown/Unknown & $\mathrm{Pl}$ & 107 & F508del/F508del & 99 & F508del/Q493X & 84 \\
\hline Unknown/Unknown & PI & 80 & F508del/621 + 1G > T & 45 & F508del/F508del & - \\
\hline Unknown/Unknown & $\mathrm{Pl}$ & 75 & F508del/621 + 1G $>T$ & 92 & F508del/R1162X & 79 \\
\hline \multicolumn{7}{|l|}{ NBS Positive (LD-NBS-pos) } \\
\hline \multicolumn{7}{|l|}{ Initial $\mathbf{S C}<30 \mathrm{mmol} / \mathrm{L}$} \\
\hline F508del/S945L & PS & $29(100)$ & F508del/G551D & 85 & F508del/Unknown & 65 \\
\hline F508del/3849 + 10kbC > T & PS & $13(44)$ & F508del/R117H & 68 & F508del/F508del & - \\
\hline \multicolumn{7}{|l|}{ Initial SC $30-59 \mathrm{mmol} / \mathrm{L}$} \\
\hline F508del/P67L ${ }^{\dagger}$ & $\mathrm{PI}$ & 52 & F508del/F508del & 95 & F508del/F508del & - \\
\hline F508del/S945L ${ }^{\dagger}$ & PS & $44(46)$ & G542X/S945L & 51 & F508del/c.234delC & 140 \\
\hline F508del/5T & PS & $36(67)$ & G542X/S945L & 74 & F508del/G551D & 105 \\
\hline F508del/ $5 T^{\dagger}$ & - & $42(72)$ & F508del/F508del & - & F508del/394delTT & - \\
\hline
\end{tabular}

$P F$, pancreatic function.

CF-causing mutations as defined by CFTR2 ${ }^{26}$ are highlighted in bold font, those with varying clinical consequence are in bold-italics, and those with uncertain significance are underlined. Italicized mutations were identified in the CFTR1 ${ }^{27}$ database only. All cases of LD-CF were matched for pancreatic exocrine status and those with unknown status were matched with PI controls.

*Patients born during IRT/IRT algorithm period.

†Patients initially classified as CFSPID.

Unavailable data indicated with a '-'.

[0.12], respectively; $P=.7)$ were evident. The LD-NBS-neg cohort had significantly lower mean height $(-0.79[0.22]$ vs -0.08 [0.14]; $P=.008)$ and weight $(-0.60[0.21]$ vs -0.06 [0.14]; $P=.03) z$-scores than NBS-CF, whereas the LD-NBS-pos cohort had a significantly lower mean BMI $z$-score than NBS-CF controls $(0.76$ [0.12] vs 1.36 [0.11]; $P=.003)$. Regarding lung function testing, 149 measurements were recorded from 25 of the patients with LD-CF and 308 measurements were recorded from 57 of the patients with NBS-CF. Given the earliest lung function testing is performed at is 6 years of age, 15 of the 45 patients with LD-CF born between 2004 and 2010 did not have testing performed. Mean (SD) lung function variables were significantly worse for LD-CF compared with NBS-CF, including forced vital capacity $(2.1 \mathrm{~L}[0.10]$ vs $2.5 \mathrm{~L}$ [0.06], respectively; $P=.003)$, forced expiratory volume in 1 second $(1.7 \mathrm{~L}[0.08]$ vs $2.0 \mathrm{~L}$ [0.06] respectively; $P=.003$ ), and forced expiratory 

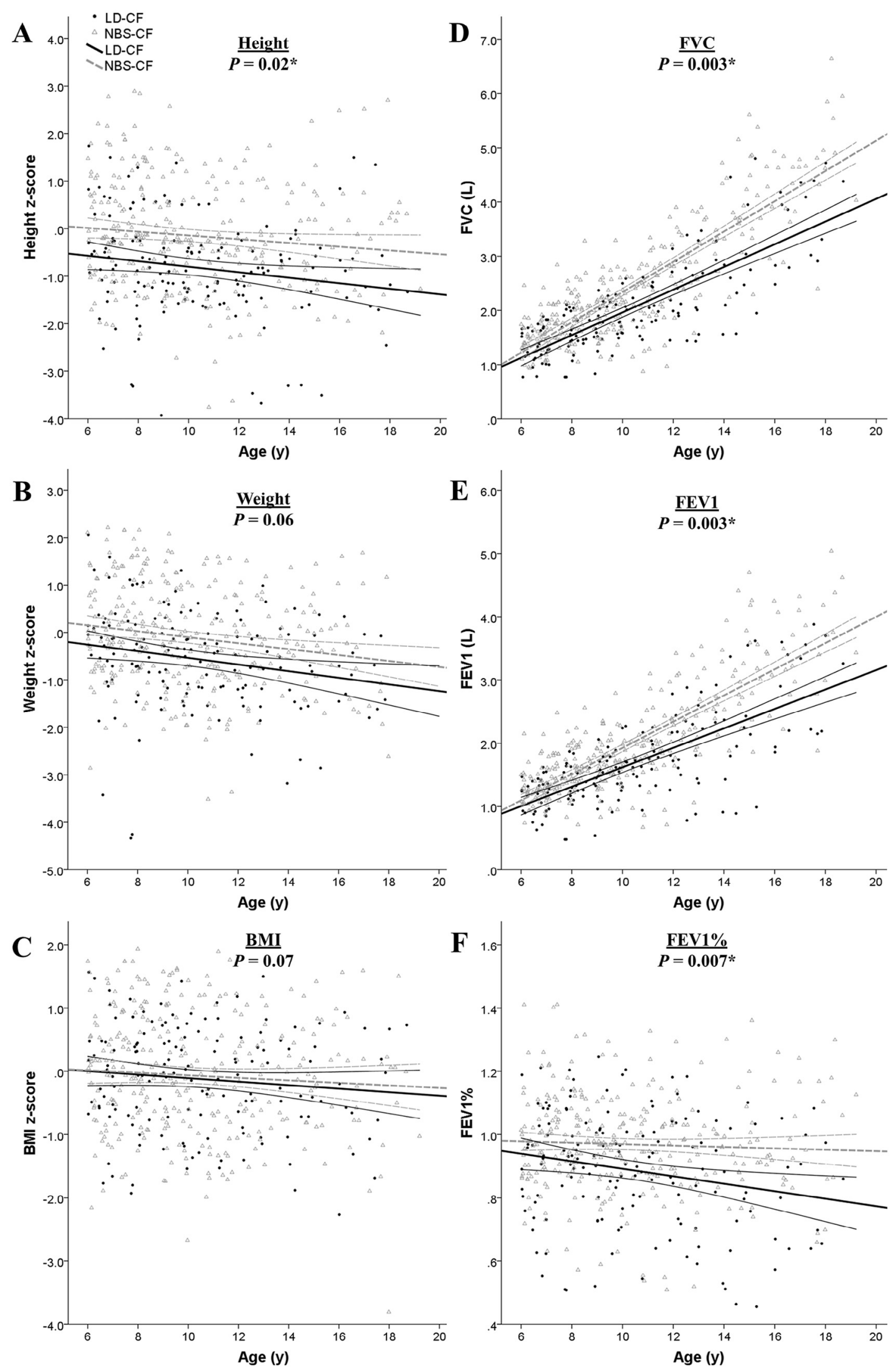

Figure 2. All recorded growth and lung function variables for all patients with LD-CF (circles) and all patients with NBS-CF (triangles). Lines represent mean (center lines; LD-CF is solid black and NBS-CF is dashed grey) with standard deviation bars (outside lines). FEV1, forced expiratory volume in 1 second; FVC, forced vital capacity. 
volume in 1 second percentage of predicted (FEV1\%) $(0.88$ [0.03] vs 0.97 [0.02], respectively; $P=.007$ ). Subset analysis revealed worse FEV1\% for both the LD-NBS-neg (0.88 [0.03] vs 0.98 [0.02]; $P=.01)$ and LD-NBS-pos $(0.86[0.02]$ vs 0.93 $[0.01] ; P=.002)$ cohorts compared with NBS-CF-matched controls.

Microbiology data from sputum and/or bronchoscopy cultures were recorded for all patients with LD-CF and patients with NBS-CF (Table III). A significantly higher rate of isolation of $P$ aeruginosa over the duration of follow-up was identified in the LD-CF cohort compared with the NBS-CF cohort ( $82 \%$ vs $69 \%$, respectively; $P=.03$ ); however, there was no difference in the age of first isolation (5.1 years [2.8-8.3] vs 4.4 years [1.5-6.9], respectively; $P=.3$ ). A significantly higher proportion of patients with LD-CF had chronic colonization with $P$ aeruginosa (47\% vs $24 \% ; P=.01$ ) and isolation of mucoid $P$ aeruginosa ( $42 \%$ vs $20 \% ; P=.008$ ). The isolation rate of $P$ aeruginosa was not different for the LD-NBS-neg cohort compared with NBS-CF cohort (79\% vs $68 \%$, respectively; $P=.3$ ); however, it was significantly higher for the 6 patients who were LD-NBS-pos ( $100 \%$ vs $33 \%$; $P=.01)$ when compared with NBS-CF matched controls. Mucoid $P$ aeruginosa isolation rates were significantly higher for the LD-NBS-neg cohort ( $44 \%$ vs $22 \% ; P=.02$ ) but not for the LD-NBS-pos cohort ( $33 \%$ vs $8 \% ; P=.2$ ) when compared with NBS-CFmatched controls. High isolation rates of $S$ aureus were also identified in both the LD-CF and NBS-CF cohorts (73\% vs $76 \%$ respectively; $P=.8$ ); however, there was no difference in age of first isolation (4.0 years [2.4-5.4] vs 2.2 years [0.75.7], respectively; $P=.1$ ) or chronic colonization ( $27 \%$ vs $34 \%$; $P=.4)$.

\section{Discussion}

In this study, we report the differences in outcomes of children with a LD-CF in the NSW NBS era. Little is known about patients with LD-CF and they represent an important subset of patients with CF. Children with LD-CF, who mostly received a false negative NBS result, had worse respiratory outcomes including poorer lung function, higher rates of chronic $P$ aeruginosa colonization, and increased frequency of hospitalization for CF-related respiratory illness at follow-up when compared with matched controls.

From 1988 to 2010, the estimated incidence of CF in NSW was 1 in 2200 live births, which is comparable with data from Victoria, Australia. ${ }^{6}$ This study calculated the incidence of $\mathrm{LD}$ CF, which occurred in 1 in 45000 live births, or alternatively 1 in every 21 patients with CF. It should be noted that the sensitivity and specificity of different CF NBS programs varies throughout the world ${ }^{8}$ and screening algorithms have been revised over time to improve detection. ${ }^{29,30}$ The findings of this study should not be taken to mean that sensitivity should be improved above all else, especially at the expense of specificity. This is particularly relevant in light of the issues related to the identification of infants with a positive NBS result but an inconclusive diagnosis of CF (CFSPID or CFTR-related metabolic syndrome). ${ }^{23,31}$ Of the 39 patients missed by NBS in our study, $22(56 \%)$ had IRT levels that were not increased and 17 (44\%) were not carriers for F508del. Of the 22 patients missed by IRT testing, 5 (23\%) were homozygous for F508del and 12 (55\%) were PI (Table II). The reason for patients with 2 severe mutations (without MI) not having an

Table III. Microbiology data

\begin{tabular}{|c|c|c|c|c|c|}
\hline \multirow[b]{2}{*}{ Variables } & \multicolumn{2}{|c|}{ LD-CF } & \multicolumn{2}{|c|}{ NBS-CF } & \multirow[b]{2}{*}{$P$} \\
\hline & $\mathbf{n}$ & Result & $\mathbf{n}$ & Result & \\
\hline \multicolumn{6}{|l|}{$P$ aeruginosa } \\
\hline Isolation & 45 & $37(82 \%)$ & 90 & $57(63 \%)$ & .03 \\
\hline Age first isolation (y) & 37 & $5.1(2.8-8.3)$ & 57 & $4.4(1.5-6.9)$ & .3 \\
\hline Chronic colonization* & 45 & $21(47 \%)$ & 90 & $22(24 \%)$ & .01 \\
\hline Age first chronic colonization (y) & 21 & $6.3(0.9)$ & 22 & $7.2(0.8)$ & .7 \\
\hline $3 \times$ cultures $^{\dagger}$ & 45 & $8(18 \%)$ & 90 & $13(14 \%)$ & .6 \\
\hline Age first $3 \times$ cultures $^{*}(\mathrm{y})$ & 8 & $7.3(1.1)$ & 13 & $7.9(1.1)$ & .7 \\
\hline \multicolumn{6}{|l|}{$P$ aeruginosa (mucoid) } \\
\hline Isolation & 45 & $19(42 \%)$ & 90 & $18(20 \%)$ & .008 \\
\hline Age first isolation (y) & 19 & $6.4(0.7)$ & 18 & $7.5(0.8)$ & .3 \\
\hline \multicolumn{6}{|l|}{$P$ aeruginosa (non-mucoid) } \\
\hline Isolation & 45 & $19(42 \%)$ & 90 & $39(43 \%)$ & .99 \\
\hline Age first isolation (y) & 19 & $4.7(0.5)$ & 39 & $5.7(0.6)$ & .3 \\
\hline \multicolumn{6}{|l|}{$P$ aeruginosa (other) } \\
\hline Isolation & 45 & $13(29 \%)$ & 90 & $27(30 \%)$ & .99 \\
\hline Age first isolation (y) & 13 & $4.5(0.9)$ & 27 & $5.0(0.8)$ & .9 \\
\hline \multicolumn{6}{|l|}{$S$ aureus } \\
\hline Isolation & 45 & $33(73 \%)$ & 90 & $68(76 \%)$ & .8 \\
\hline Age first isolation (y) & 33 & $4.0(2.4-5.4)$ & 68 & $2.2(0.7-5.7)$ & .1 \\
\hline Chronic colonization* & 45 & $12(27 \%)$ & 90 & $31(34 \%)$ & .4 \\
\hline Age first $3 \times$ cultures $^{*}(\mathrm{y})$ & 12 & $7.7(1.0)$ & 31 & $8.3(0.8)$ & .7 \\
\hline MRSA & 45 & $1(2 \%)$ & 90 & $12(13 \%)$ & .06 \\
\hline
\end{tabular}

Data presented as number (percentage), mean (SD), or median (IQR)

${ }^{*}$ Chronic colonization defined by 3 consecutive cultures within a 12-month period or isolation of mucoid Pseudomonas spp.

$\uparrow 3 \times$ cultures, 3 consecutive cultures. 
elevated IRT remains unclear. Six of the patients with LD-CF were born during the IRT/IRT screening protocol and 1 of these would have been detected by the subsequent IRT/DNA protocol.

Late diagnosis patients with CF had significantly worse health at time of diagnosis, including more respiratory $(P<.0001)$, gastrointestinal $(P=.005)$, and failure-to-thrive manifestations $(P<.0001)$, which is not surprising because they were likely the cause(s) for clinical attention. Subset analysis also revealed that patients who are LD-NBS-neg and patients who are LD-NBS-pos have significantly more respiratory manifestations than NBS-CF matched controls $(P<.0001$ and $P=.02$, respectively). In the era of CFTR-targeted therapies, ${ }^{32,33}$ early diagnosis and rescue therapy before irreversible organ damage occurs (ie, pancreatic atrophy in utero ${ }^{34}$ and within months postnatally ${ }^{35}$ leading to organ failure and becoming PI) becomes increasingly important.

To date, several studies support the notion that NBS protocols reduce the therapeutic burden, hospitalization rates, and morbidity associated with $\mathrm{CF}^{36-41}$; however, sparing the Wisconsin CF Study Group, high-quality, randomized, controlled trials are lacking. ${ }^{11}$ Our study provides further support for NBS with LD-CF having a significantly higher median number of hospital admissions per year for CF-related respiratory illness compared with NBS-CF controls ( 0.49 vs 0.20 , respectively; $P=.0004$ ).

Waters et $\mathrm{al}^{15}$ in 1999 found that in the 3 years before and after the implementation of NBS in NSW, NBS was associated with greater height $z$-scores at 5 years of age and improved FEV $1 \%$ at 5 and 10 years of age. The most convincing evidence regarding the nutritional and growth benefits of NBS come from the Wisconsin CF randomized longitudinal studies. ${ }^{12,42}$

Several other studies comparing historical cohorts with NBS have also shown nutritional and/or respiratory benefits. ${ }^{16,18,43,44}$ Interestingly, in our study, the mixed models analysis for height $z$-scores of patients with LD-CF was significantly lower than NBS-CF controls $(-0.65[0.22]$ vs -0.03 [0.15], respectively; $P=.02)$. Although not significant, the LD-CF also had a trend toward lower weight $z$-scores and BMI compared with NBSCF. The LD-NBS-neg cohort, however, had both lower height $(P=.008)$ and weight $(P=.03) z$-scores compared with matched NBS-CF controls. Although the numbers were small $(n=6)$, patients who were LD-NBS-pos had significantly lower BMI scores over the duration of follow-up compared with NBSCF controls $(P=.003)$.

One of the strengths of this study is the use of the mixed models analysis of lung function, which takes into account all recorded measures for each individual patient over their entire duration of follow-up. The LD-CF cohort had a significantly worse mean FEV1\% compared with NBS-CF controls (0.88 vs 0.98 , respectively; $P=.01$ ). This result was also evident in both the LD-NBS-neg and LD-NBS-pos subset cohorts (when compared with their respective NBS-CF control cohorts). Although the association between NBS and improved longterm lung function has been limited, ${ }^{18,42}$ this finding has previously been reported in our NSW cohort. ${ }^{18,21}$ The worse lung function in the LD-CF cohort is probably associated with the significantly higher rate of mucoid $P$ aeruginosa isolation compared with NBS-CF controls ( $42 \%$ vs $20 \%$; $P=.008)$. Although a proportion of the LD-NBS-neg cohort have a severe phenotype (ie, probably PI at birth and thus lower IRT) this alone does not account for their worse lung function, because they also have higher isolation rates of mucoid $P$ aeruginosa compared with matched NBS-CF controls ( $44 \%$ vs $22 \%$, respectively; $P=.02$ ). The remaining 6 patients who were LDNBS-pos who are predominantly PS, also had a higher rate of mucoid $P$ aeruginosa (33\% vs $8 \%$ ), but this failed to attain significance $(P=.2)$, which may be owing to the sample size. Isolation of $P$ aeruginosa and in particular chronic or mucoid $P$ aeruginosa infection has long been associated with worse lung function and imaging scores in patients with $\mathrm{CF}^{45-47} \mathrm{~A}$ recent multivariable model from the Wisconsin group identified several modifiable extrinsic risk factors for the progression of lung disease in children with CF, which included colonization with mucoid $P$ aeruginosa. ${ }^{48}$ Early identification and eradication is likely to have long-lasting impacts in children with $\mathrm{CF},{ }^{4,50}$ as are preventative measures. ${ }^{51,52}$

The intriguing group of patients who are screened positive and have sweat tests with an initially normal $(<30 \mathrm{mmol} /$ $\mathrm{L})$ or intermediate SC $(30-59 \mathrm{mmol} / \mathrm{L})$ result are highlighted in this study. Of the 4 patients with CF initially identified as CFSPID (Table II), 2 carried 2 disease-causing mutations (F508del/P67L and F508del/S945L) with 1 being PI (F508del/ $P 67 L$ ) at most recent review (a small but important finding that differs from previous reports ${ }^{53}$ ). Interestingly, the $2 \mathrm{pa}-$ tients with CFSPID with a $5 T$ mutation (which is thought to be of variable clinical significance $\left.{ }^{26}\right)$ progressed to having a positive SC result $(\geq 60 \mathrm{mmol} / \mathrm{L})$. Table II also includes $2 \mathrm{pa}$ tients with an initially normal SC $(<30 \mathrm{mmol} / \mathrm{L})$ who were heterozygotes for F508del and a PS mutation, S945L and $3849+10 \mathrm{kbC}>T \cdot{ }^{26} \mathrm{~A}$ concerning finding for the patients who were LD-NBS-pos was that they all had $P$ aeruginosa isolated, and this parallels previous reports. ${ }^{53-56}$

This study is limited by the small sample size; however, it is also reassuring that the number of patients with $\mathrm{LD}-\mathrm{CF}$ is small and the NBS program performs well. The optimal NBS program for $\mathrm{CF}$ is yet to be determined ${ }^{57}$ and, as adjuncts to the NSW program, both positive family history and MI protocols have been developed; however, the impact of these measures are yet to be evaluated. Further limitations include the retrospective nature and the number of patients $<6$ years of age by 2010 who were too young to undergo lung function testing. Not all patients had extensive genotyping or gene sequencing performed; thus, not all CFTR mutations were identified. The documented phenomenon of patients progressing from $\mathrm{PS}$ to $\mathrm{PI}^{58}$ also provides a limitation. Patients who are yet to receive a LD-CF and those who have done so after transitioning to adult care (in Australia, data are sent voluntarily to the national CF registry) may also be missed by this analysis. The ever-expanding list of disease-causing mutations provides further limitations. NBS and this study are not designed to capture those patients who also lie on the spectrum of CFTR dysfunction including those with CFTRrelated disorders such as congenital bilateral absence of the vas 
deferens or idiopathic recurrent/chronic pancreatitis, ${ }^{59,60}$ both of which are more likely to present in adult life. In addition, the heterogeneity of the LD-CF cohort made matching with NBS-CF controls challenging. Despite these challenges, the SC levels were not different between the LD-CF and NBS-CF cohorts ( 83.2 vs 89.1 , respectively; $P=.2$ ). This finding, along with the matching of pancreatic function status, helps to control for the differences in initial presentation and the potential effect of improvement over time. Furthermore, matching for CF clinic aimed to reduce any variance in clinical practice. The strength of this study lies in the 2 decades of NBS experience. Although problematic to perform, long-term prospective studies may provide more insight into those patients who are missed by NBS and receive a LD-CF.

In conclusion, this study serves to highlight the outcomes of those patients with CF who have a missed or late diagnosis despite NBS. Patients who receive a late diagnosis seem to be different genotypically and phenotypically from patients with NBS-CF, as are the differences for those who are LD-NBSneg and LD-NBS-pos. A late diagnosis seems to be associated with worse health at diagnosis and worse outcomes with regard to growth and respiratory outcomes (FEV1\% and $P$ aeruginosa colonization). Coupled with emerging CFTRtargeted therapies, these findings provide further support for NBS programs for CF.

We thank Geoff Sims (Australian CF Registry Database) for his assistance in data acquisition.

Submitted for publication Jun 4, 2016; last revision received Sep 7, 2016; accepted Oct 12, 2016

Reprint requests: (Keith) Chee Y. Ooi, MBBS, FRACP, PhD, Sydney Children's Hospital, School of Women's and Children's Health, High Street, Randwick, NSW 2031, Australia. E-mail: keith.ooi@unsw.edu.au

\section{References}

1. Massie RJ, Olsen M, Glazner J, Robertson CF, Francis I. Newborn screening for cystic fibrosis in Victoria: 10 years' experience (1989-1998). Med J Aust 2000;172:584-7.

2. Parad RB, Comeau AM. Newborn screening for cystic fibrosis. Pediatr Ann 2003;32:528-35.

3. Comeau AM, Parad RB, Dorkin HL, Dovey M, Gerstle R, Haver K, et al. Population-based newborn screening for genetic disorders when multiple mutation DNA testing is incorporated: a cystic fibrosis newborn screening model demonstrating increased sensitivity but more carrier detections. Pediatrics 2004;113:1573-81.

4. Sontag MK, Hammond KB, Zielenski J, Wagener JS, Accurso FJ. Twotiered immunoreactive trypsinogen-based newborn screening for cystic fibrosis in Colorado: screening efficacy and diagnostic outcomes. J Pediatr 2005; 147:S83-8.

5. Riordan JR, Rommens JM, Kerem B, Alon N, Rozmahel R, Grzelczak Z, et al. Identification of the cystic fibrosis gene: cloning and characterization of complementary DNA. Science (New York, NY) 1989;245:106673.

6. Massie RJ, Curnow L, Glazner J, Armstrong DS, Francis I. Lessons learned from 20 years of newborn screening for cystic fibrosis. Med J Aust 2012;196:67-70.

7. Rock MJ, Hoffman G, Laessig RH, Kopish GJ, Litsheim TJ, Farrell PM. Newborn screening for cystic fibrosis in Wisconsin: nine-year experience with routine trypsinogen/DNA testing. J Pediatr 2005;147:S73-7.

8. Gonska T, Ratjen F. Newborn screening for cystic fibrosis. Expert Rev Respir Med. 2015;9:619-31.
9. Wilcken B, Brown AR, Urwin R, Brown DA. Cystic fibrosis screening by dried blood spot trypsin assay: results in 75,000 newborn infants. J Pediatr 1983;102:383-7.

10. Wilcken B, Wiley V, Sherry G, Bayliss U. Neonatal screening for cystic fibrosis: a comparison of two strategies for case detection in 1.2 million babies. J Pediatr 1995; 127:965-70.

11. Southern KW, Merelle MM, Dankert-Roelse JE, Nagelkerke AD. Newborn screening for cystic fibrosis. Cochrane Database Syst Rev 2009;(1): CD001402.

12. Farrell PM, Kosorok MR, Rock MJ, Laxova A, Zeng L, Lai HC, et al. Early diagnosis of cystic fibrosis through neonatal screening prevents severe malnutrition and improves long-term growth. Wisconsin Cystic Fibrosis Neonatal Screening Study Group. Pediatrics 2001;107:1-13.

13. Farrell PM, Li Z, Kosorok MR, Laxova A, Green CG, Collins J, et al. Bronchopulmonary disease in children with cystic fibrosis after early or delayed diagnosis. Am J Respir Crit Care Med 2003;168:1100-8.

14. Wald NJ, Morris JK. Neonatal screening for cystic fibrosis. BMJ 1998;316:404-5.

15. Waters DL, Wilcken B, Irwing L, Van Asperen P, Mellis C, Simpson JM, et al. Clinical outcomes of newborn screening for cystic fibrosis. Arch Dis Child Fetal Neonatal Ed 1999;80:F1-7.

16. Merelle ME, Nagelkerke AF, Lees CM, Dezateux C. Newborn screening for cystic fibrosis. Cochrane Database Syst Rev 2001;(3):CD001402.

17. Grosse SD, Boyle CA, Botkin JR, Comeau AM, Kharrazi M, Rosenfeld M, et al. Newborn screening for cystic fibrosis: evaluation of benefits and risks and recommendations for state newborn screening programs. Morbid Mortal Recomm Rep 2004;53:1-36.

18. McKay KO, Waters DL, Gaskin KJ. The influence of newborn screening for cystic fibrosis on pulmonary outcomes in New South Wales. J Pediatr 2005; 147:S47-50.

19. Rosenthal M. Newborn screening for cystic fibrosis: the motion againstvoices in the wilderness. Paediatr Respir Rev 2008;9:295-300.

20. Farrell PM, Rosenstein BJ, White TB, Accurso FJ, Castellani C, Cutting GR, et al. Guidelines for diagnosis of cystic fibrosis in newborns through older adults: Cystic Fibrosis Foundation consensus report. J Pediatr 2008;153:S4-14.

21. Dijk FN, McKay K, Barzi F, Gaskin KJ, Fitzgerald DA. Improved survival in cystic fibrosis patients diagnosed by newborn screening compared to a historical cohort from the same centre. Arch Dis Child 2011;96:1118-23.

22. Massie RJ, Wilcken B, Van Asperen P, Dorney S, Gruca M, Wiley V, et al. Pancreatic function and extended mutation analysis in DeltaF508 heterozygous infants with an elevated immunoreactive trypsinogen but normal sweat electrolyte levels. J Pediatr 2000;137:214-20.

23. Borowitz D, Parad RB, Sharp JK, Sabadosa KA, Robinson KA, Rock MJ, et al. Cystic Fibrosis Foundation practice guidelines for the management of infants with cystic fibrosis transmembrane conductance regulatorrelated metabolic syndrome during the first two years of life and beyond. J Pediatr 2009;155:S106-16.

24. Mayell S. Management of equivocal diagnosis, the European consensus project. In: European Cystic Fibrosis Conference 2014. June 13, 2014; Gothenburg, Sweden.

25. Waite RD, Wareham DW, Gardiner S, Whiley RA. A simple, semiselective medium for anaerobic isolation of anginosus group streptococci from patients with chronic lung disease. J Clin Microbiol 2012;50:14302 .

26. Sosnay PR, Siklosi KR, Van Goor F, Kaniecki K, Yu H, Sharma N, et al. Defining the disease liability of variants in the cystic fibrosis transmembrane conductance regulator gene. Nat Genet 2013;45:1160-7.

27. Cystic Fibrosis Mutation Database (CFTR1). www.genet.sickkids.on.ca/. 2011; Accessed January 12, 2016.

28. Pressler T, Bohmova C, Conway S, Dumcius S, Hjelte L, Hoiby N, et al. Chronic Pseudomonas aeruginosa infection definition: EuroCareCF Working Group report. J Cyst Fibros 2011;10(Suppl 2):S75-8.

29. Kloosterboer M, Hoffman G, Rock M, Gershan W, Laxova A, Li Z, et al. Clarification of laboratory and clinical variables that influence cystic fibrosis newborn screening with initial analysis of immunoreactive trypsinogen. Pediatrics 2009;123:e338-46. 
30. Sanders DB, Lai HJ, Rock MJ, Farrell PM. Comparing age of cystic fibrosis diagnosis and treatment initiation after newborn screening with two common strategies. J Cyst Fibros 2012;11:150-3.

31. Munck A, Mayell SJ, Winters V, Shawcross A, Derichs N, Parad R, et al. Cystic Fibrosis Screen Positive, Inconclusive Diagnosis (CFSPID): a new designation and management recommendations for infants with an inconclusive diagnosis following newborn screening. J Cyst Fibros. 2015;14:706-13.

32. Ramsey BW, Davies J, McElvaney NG, Tullis E, Bell SC, Drevinek P, et al. A CFTR potentiator in patients with cystic fibrosis and the G551D mutation. N Engl J Med 2011;365:1663-72.

33. Boyle MP, Bell SC, Konstan MW, McColley SA, Rowe SM, Rietschel E, et al. A CFTR corrector (lumacaftor) and a CFTR potentiator (ivacaftor) for treatment of patients with cystic fibrosis who have a phe508del CFTR mutation: a phase 2 randomised controlled trial. Lancet Respir Med 2014;2:527-38.

34. Imrie JR, Fagan DG, Sturgess JM. Quantitative evaluation of the development of the exocrine pancreas in cystic fibrosis and control infants. Am J Pathol 1979;95:697-707.

35. Couper RT, Corey M, Durie PR, Forstner GG, Moore DJ. Longitudinal evaluation of serum trypsinogen measurement in pancreatic-insufficient and pancreatic-sufficient patients with cystic fibrosis. J Pediatr 1995; 127:408-13.

36. McKay K, Wilcken B. Newborn screening for cystic fibrosis offers an advantage over symptomatic diagnosis for the long term benefit of patients: the motion for. Paediatr Respir Rev 2008;9:290-4.

37. Wilcken B, Chalmers G. Reduced morbidity in patients with cystic fibrosis detected by neonatal screening. Lancet (London, England) 1985;2:1319-21.

38. Dankert-Roelse JE, te Meerman GJ, Martijn A, ten Kate LP, Knol K. Survival and clinical outcome in patients with cystic fibrosis, with or without neonatal screening. J Pediatr 1989;114:362-7.

39. Siret D, Bretaudeau G, Branger B, Dabadie A, Dagorne M, David V, et al. Comparing the clinical evolution of cystic fibrosis screened neonatally to that of cystic fibrosis diagnosed from clinical symptoms: a 10-year retrospective study in a French region (Brittany). Pediatr Pulmonol 2003;35:342-9.

40. Accurso FJ, Sontag MK, Wagener JS. Complications associated with symptomatic diagnosis in infants with cystic fibrosis. J Pediatr 2005;147:S3741.

41. Sims EJ, McCormick J, Mehta G, Mehta A. Newborn screening for cystic fibrosis is associated with reduced treatment intensity. J Pediatr 2005; 147:306-11.

42. Farrell PM, Kosorok MR, Laxova A, Shen G, Koscik RE, Bruns WT, et al. Nutritional benefits of neonatal screening for cystic fibrosis. Wisconsin Cystic Fibrosis Neonatal Screening Study Group. N Engl J Med 1997;337:963-9.

43. Dankert-Roelse JE, te Meerman GJ. Long term prognosis of patients with cystic fibrosis in relation to early detection by neonatal screening and treatment in a cystic fibrosis centre. Thorax 1995;50:712-8.

44. Mastella G, Zanolla L, Castellani C, Altieri S, Furnari M, Giglio L, et al. Neonatal screening for cystic fibrosis: long-term clinical balance. Pancreatology 2001;1:531-7.
45. Kosorok MR, Zeng L, West SE, Rock MJ, Splaingard ML, Laxova A, et al. Acceleration of lung disease in children with cystic fibrosis after Pseudomonas aeruginosa acquisition. Pediatr Pulmonol 2001;32:27787.

46. Li Z, Kosorok MR, Farrell PM, Laxova A, West SE, Green CG, et al. Longitudinal development of mucoid Pseudomonas aeruginosa infection and lung disease progression in children with cystic fibrosis. JAMA 2005;293:581-8.

47. Schaedel C, de Monestrol I, Hjelte L, Johannesson M, Kornfalt R, Lindblad A, et al. Predictors of deterioration of lung function in cystic fibrosis. Pediatr Pulmonol 2002;33:483-91.

48. Sanders DB, Li Z, Laxova A, Rock MJ, Levy H, Collins J, et al. Risk factors for the progression of cystic fibrosis lung disease throughout childhood. Ann Am Thorac Soc 2014;11:63-72.

49. Mayer-Hamblett N, Kronmal RA, Gibson RL, Rosenfeld M, RetschBogart G, Treggiari MM, et al. Initial Pseudomonas aeruginosa treatment failure is associated with exacerbations in cystic fibrosis. Pediatr Pulmonol 2012;47:125-34.

50. Treggiari MM, Retsch-Bogart G, Mayer-Hamblett N, Khan U, Kulich M, Kronmal R, et al. Comparative efficacy and safety of 4 randomized regimens to treat early Pseudomonas aeruginosa infection in children with cystic fibrosis. Arch Pediatr Adolesc Med. 2011;165:84756 .

51. Baumann U, Stocklossa C, Greiner W, von der Schulenburg JM, von der Hardt H. Cost of care and clinical condition in paediatric cystic fibrosis patients. J Cyst Fibros 2003;2:84-90.

52. Hoiby N, Frederiksen B, Pressler T. Eradication of early Pseudomonas aeruginosa infection. J Cyst Fibros 2005;4(Suppl 2):49-54.

53. Ooi CY, Castellani C, Keenan K, Avolio J, Volpi S, Boland M, et al. Inconclusive diagnosis of cystic fibrosis after newborn screening. Pediatrics 2015;135:e1377-85.

54. Ren CL, Fink AK, Petren K, Borowitz DS, McColley SA, Sanders DB, et al. Outcomes of infants with indeterminate diagnosis detected by cystic fibrosis newborn screening. Pediatrics 2015;135:e1386-92.

55. Groves T, Robinson P, Wiley V, Fitzgerald DA. Long-term outcomes of children with intermediate sweat chloride values in infancy. J Pediatr 2015;166:1469-74, e1-3.

56. Ren CL, Desai H, Platt M, Dixon M. Clinical outcomes in infants with cystic fibrosis transmembrane conductance regulator (CFTR) related metabolic syndrome. Pediatr Pulmonol 2011;46:1079-84.

57. Castellani C, Southern KW, Brownlee K, Dankert Roelse J, Duff A, Farrell $\mathrm{M}$, et al. European best practice guidelines for cystic fibrosis neonatal screening. J Cystic Fibros. 2009;8:153-73.

58. Cipolli M, Castellani C, Wilcken B, Massie J, McKay K, Gruca M, et al. Pancreatic phenotype in infants with cystic fibrosis identified by mutation screening. Arch Dis Child 2007;92:842-6.

59. Ooi CY, Dupuis A, Ellis L, Jarvi K, Martin S, Ray PN, et al. Does extensive genotyping and nasal potential difference testing clarify the diagnosis of cystic fibrosis among patients with single-organ manifestations of cystic fibrosis? Thorax 2014;69:254-60.

60. Ooi CY, Dupuis A, Ellis L, Jarvi K, Martin S, Gonska T, et al. Comparing the American and European diagnostic guidelines for cystic fibrosis: same disease, different language? Thorax 2012;67:618-24. 


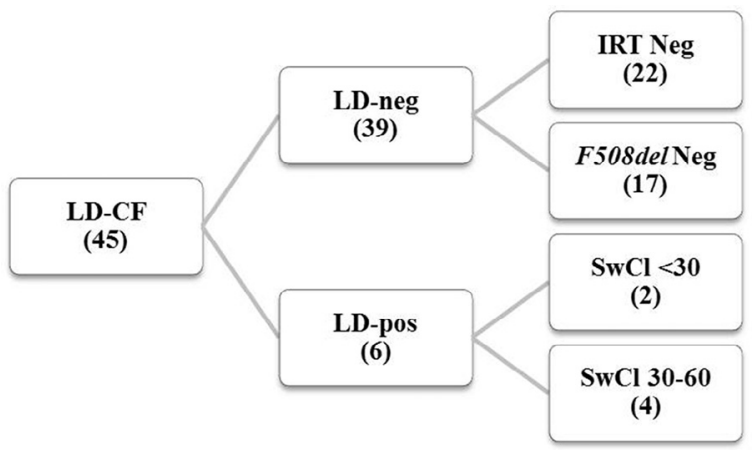

Figure 1. Distribution of LD-CF cases. $\mathrm{SwCl}$, sweat chloride. 